\title{
Period Doubling Phenomenon as the Origin of Electron Properties
}

\author{
Ari Lehto \\ Aalto University, Espoo, Finland \\ cwmies@gmail.com
}

\begin{abstract}
It is proposed that the electrons have an intrinsic periodic property, which determines particle's rest energy, electric charge, and magnetic moment. Numerical analysis shows that the correct periods are generated by a precise period doubling cascade starting at the Planck scale. Periods corresponding to the values of the intrinsic physical properties of the electron and positron belong to a subset of stable periods. The periodic structures of the rest energy and magnetic moment consist of three internal degrees of freedom, whereas the Coulomb energy of the electric charge consists of four. The number of period doublings for the elementary charge determines the value of the fine structure constant alpha.
\end{abstract}

Keywords: period doubling, electron structure, elementary charge, electron magnetic moment, fine structure constant, Planck scale

\section{INTRODUCTION}

The purpose of this article is to give attention to the possibility that the per se well known physical phenomenon, namely the period doubling process occurring in various nonlinear dynamical systems [1-6], can be directly related to the physical properties of matter.

The possible existence at the Planck scale of the period doubling phenomenon was discovered by experimental data analysis showing regular discrete peaks [7] interpreted as degrees of freedom.

In this article the properties of the electron-positron pair and the fine structure constant are numerically analyzed in Chapter 2 by using the simple period doubling formulas presented in Chapter 1 for three and four degrees of freedom.

Louis de Broglie [8] in 1924 proposed that particles behave as a wave with an associated wavelength $\lambda=h / p$, where $p$ is the momentum of the particle and $h$ the Planck constant. The Compton wavelength $\lambda=h / m c$, where $m$ is the rest mass of the particle, corresponds to the rest momentum, or rest energy, of the particle.
George Thomson [9], Clinton Davisson and Lester Germer [10] verified the wave behavior of electrons in diffraction experiments in the nineteen twenties. The matter wave concept led to the invention and development of quantum mechanics by $\mathrm{E}$. Schrödinger, W. Heisenberg, W. Pauli, M. Born and others. The physical nature of the matter wave has remained obscure, however.

The wave nature of matter raises the question whether the electron could possess a periodic structure related to the Compton wavelength. This question is especially intriguing because the electron is traditionally considered as pointlike and structureless.

It is suggested that such a periodic structure exists and that the structure creating process is the wellknown period doubling cascade taking place in nonlinear dynamical systems, and that the Planck time can be taken as the fundamental period.

The Planck units, defined by natural constants, have been of interest to both cosmologists and the elementary particle physicists as a possible base for fundamental quantum physical phenomena.

However, the Planck units are far too large or too small to be meaningful e.g. in connection with the 
properties of an electron. For instance, the Compton wavelength of the Planck mass is c. $10^{-35} \mathrm{~m}$, which can be compared with that of an electron, which is $\mathrm{c}$. $10^{-12} \mathrm{~m}$. The value of the Planck charge is not too far from the elementary charge, however.

Period doubling is a universal property of nonlinear dynamical systems, as shown by M. Feigenbaum [10]. If the natural constants are the same everywhere, then the Planck units can be used as a universal basis. The Planck energy, magnetic moment, and the Planck charge, listed in Table 1, have no correspondence with the real world. The Planck units have been defined here by $h$ (not $h$-bar), because energy is $E=h f=h /$ period . The definitions of the relevant Planck units are shown in equations (1) to (4), where $h$ is the Planck constant, $G$ the gravitational constant, $c$ the speed of light, $e$ the elementary electric charge and $\mu_{o}$ the vacuum permeability.

It is convenient to define the Planck time $t_{p}\left(\approx 10^{-43}\right.$ s) as the fundamental period due to its universality, and because energy (2) and magnetic moment (3) can be derived from it.

$$
\begin{aligned}
& t_{P}=\sqrt{\frac{h G}{c^{5}}} \\
& E_{p}=\frac{h}{t_{p}} \\
& \mu_{P}=\frac{e c^{2} t_{P}}{4 \pi} \\
& q_{P}=\sqrt{\frac{4 \pi h}{\mu_{o} c}}
\end{aligned}
$$

$E_{p}$ is the Planck energy, $\mu_{p}$ the Planck magnetic moment and $q_{p}$ the Planck charge.

Table 1. Planck units and electron properties.

\begin{tabular}{ll}
\hline \hline Planck unit & Value \\
\hline Energy & $3.0604 \cdot 10^{22} \mathrm{MeV}$ \\
Magnetic moment & $1.5485 \cdot 10^{-46} \mathrm{Am}^{2}$ \\
Charge & $4.7013 \cdot 10^{-18} \mathrm{As}$ \\
\hline \hline Electron property & Value \\
\hline Rest energy & $0.51099 \quad \mathrm{MeV}$ \\
Magnetic moment & $9.2848 \cdot 10^{-24} \mathrm{Am}^{2}$ \\
Charge & $1.6022 \cdot 10^{-19} \mathrm{As}$ \\
\hline \hline
\end{tabular}

Comparison of the values shows that the electron properties are quite far away from the corresponding Planck units except for the electric charge. It is quite evident that many period doublings, or energy halvings, are needed to obtain the values for the electron properties from the corresponding Planck units.

The Planck scale magnetic moment is defined as a current loop $\mu_{p}=i A$, where the current $i$ is the elementary electric charge divided by the Planck time (=period). The loop area is $A=\pi r^{2}$, where $r=c t_{p} / 2 \pi$ i.e. the circumference of the loop is the Planck length and radius the Planck length over $2 \pi$, resulting in equation (3).

If an electron related period $t_{n}$ results from a onedegree-of-freedom period doubling process at the Planck scale, then

$$
t_{n}=2^{n} t_{p}
$$

where $t_{p}$ is the Planck time (period) and $n$ the number of period doublings.

Equation (6) displays the normalized $t_{n}$, i.e. $t_{n}$ is presented as ratio to the reference value.

$$
\frac{t_{n}}{t_{p}}=2^{n}
$$

Consider a system with several degrees of freedom, each having a different number of period doublings. For a system with three periods $t_{i}, t_{j}$ and $t_{k}$ the corresponding geometric phase-space shape is a straight parallelepiped, whose volume is $V_{i j k}=t_{i} t_{j} t_{k}$. The volume doubles if any one period doubles. If this structure represents e.g. an elementary particle, one should, in principle, be able to observe the individual periods. Unfortunately, the particle collision and decay experiments do not separate the degrees of freedom from one another but return the particle rest energy $E=h / t_{c}$ corresponding to one characteristic period $t_{c}$ according to (2).

Conservation of energy requires that

$$
t_{c}{ }^{3}=t_{i} t_{j} t_{k}
$$

which means that 


$$
t_{c}=\left(t_{i} t_{j} t_{k}\right)^{\frac{1}{3}}
$$

and so

$$
\frac{t_{c}}{t_{p}}=2^{n}=2^{\frac{i+j+k}{3}}=2^{\frac{N}{3}}
$$

where $N=i+j+k$ is the total number of period doublings. The exponent of 2 in (9) is an integer divided by the number of the degrees of freedom i.e. cube root of $2^{N}$.

For an observer, energy is a scalar quantity, which manifests itself as a geometric mean of the periods of the internal degrees of freedom according to (8) and (9).

It is also known that periods of the form

$$
t_{n}=2^{2^{n}} t_{o}
$$

are especially stable [10]. In (10) $t_{o}$ is the fundamental period of the system and $n$ the number of period doublings.

Numerical calculations in the next paragraph show that (9) and (10) work well with the properties of the electron-positron pair.

\section{THE ELECTRON-POSITRON PAIR}

\subsection{Numerical calculations}

The electron and positron are always born together in the pair production process assumed nonlinear. The pair is a $1.022 \mathrm{MeV}$ entity, and both $0.511 \mathrm{MeV}$ particles carry the elementary charge. Applying (9) for the rest energy $E_{e p}$ of the electron-positron pair, one obtains

$n=\frac{\log \left(\frac{E_{e p}}{E_{p}}\right)}{\log (2)}=-74.665=-\frac{223.99}{3} \cong-\frac{224}{3}$

The ratio of the pair Compton wavelength to the Planck length naturally yields the same $n$-value. The magnetic moment $\mu_{e p}$ corresponding to the pair Compton wavelength is

$$
n=\frac{\log \left(\frac{\mu_{e p}}{\mu_{p}}\right)}{\log (2)}=74.666=\frac{224}{3}
$$

where $\mu_{e p}$ is half of the electron magnetic moment (Table 1), because the current loop for the pair structure is smaller. Equations (11) and (12) show that $n$ is an integer divided by three as suggested by (9) for three degrees of freedom.

The remarkable observation is that the number of period doublings is the same for the electronpositron pair rest energy and magnetic moment albeit the fact that the Planck units have been calculated from a different set of natural constants ( $G$ in (1) and (2), $\mu_{o}$ in (4)).

The electron rest energy is half of the original pair energy. Therefore, the corresponding period for the $0.511 \mathrm{MeV}$ energy is longer by factor of 2 , and so the number of period doublings is one more, or $n=-$ $75.665 \approx-227 / 3$.

The magnetic moment corresponding to the larger loop of the electron structure is also obtained by one more period doubling, or $n=75.666=227 / 3$ period doublings.

The total number of period doublings in three degrees of freedom is $N=224$ for the pair. The number of period doublings is negative for the rest energy, because the doubling process divides the Planck energy (h/period) into precise smaller parts, while the number of doublings is positive for the magnetic moment, because the magnetic moment gets larger with longer periods according to (3).

The Planck charge is quite close to the elementary electric charge $e$. This means that the total number of period doublings for $e$ is much less than 224 . Comparison of the squares of the charges, i.e. their Coulomb energies, yields

$$
n=\frac{\log \left(\frac{e}{q_{p}}\right)^{2}}{\log (2)}=-9.7499 \cong-\frac{39}{4}
$$

The total number of period doublings is $N=39$. The fourth root indicates that the number of degrees of freedom is four and the phase space volume correspondingly $V_{l m n p}=t_{l} t_{m} t_{n} t_{p}$. The characteristic period is $t_{c}=\left(t_{l} t_{m} t_{n} t_{p}\right)^{1 / 4}$ analogously to (8). At first sight the $N$-values do not seem to be related to anything familiar. 


\subsection{Interpretation of the $N$-values}

For one degree of freedom, the $n$-values should be integers. Equations (11), (12) and (13) show that $n= \pm N / 3=224 / 3$ for the magnetic moment and the rest energy of the pair, and $n=-N / 4=-39 / 4$ for the Coulomb energy.

Mathematically speaking $N=i+j+k$ for (11) and (12) and $N=l+m+n+p$ for (13), where integers $i, j, k$ and $l, m, n, p$ represent the individual period doublings for each degree of freedom.

The electron and positron are known to be stable, and one may expect $i, j, k$ and $l, m, n, p$ to be of the form of (10). Indeed $N=i+j+k=224=32+64+128=$ $2^{5}+2^{6}+2^{7}$, and $N=l+m+n+p=39=1+2+4+32=$ $2^{0}+2^{1}+2^{2}+2^{5}$, showing that the stability condition (10) is fulfilled for each degree of freedom. Equations (12) and (13) show that the periodic nature of matter also applies to the magnetic moment and the Coulomb energy of the electric charge.

Table 2 compares measured values to those given by $n=224 / 3$ and $n=39 / 4$ in (5) for the electron-positron pair rest energy $E_{e p}$, magnetic moment $\mu_{e p}$ and elementary charge $e^{2}$ correspondingly ( $t_{p}$ replaced by $E_{p}, q_{p}$ or $\left.\mu_{p}\right)$. For convenience Table 2 shows the value of $e$ instead of $e^{2}$.

Table 2. Comparison of the calculated and measured values.

\begin{tabular}{lcc}
\hline \hline Electron & Measured & Calculated \\
\hline$E_{e}(\mathrm{MeV})$ & 0.511 & 0.511 \\
$e(\mathrm{As})$ & $-1.6022 \cdot 10^{-19}$ & $-1.6021 \cdot 10^{-19}$ \\
$\mu_{e}\left(\mathrm{Am}^{2}\right)$ & $-9.285 \cdot 10^{-24}$ & $-9.286 \cdot 10^{-24}$ \\
\hline \hline
\end{tabular}

The $N$-values for the electric charge squared differ from those of the rest energy and magnetic moment except for period $i=p=2^{5}$, which is common to all. Three degrees of freedom $(l=1, m=2, n=4)$ of the Coulomb energy are independent of the degrees of freedom of the rest energy and magnetic moment of the electron. This means that mass and electric charge related period doublings are independent phenomena ( $G$ vs. $\mu_{o}$ ).

The Planck mass-energy (2) does not contain the Coulomb-energy, which can be calculated knowing the ratio of the elementary charge squared to the Planck charge squared. The Coulomb-energy for the pair structure becomes $2^{-74.667} \cdot\left(2^{-9.75} \cdot E_{p}\right)=0.00119$ $\mathrm{MeV}$ and $0.00059 \mathrm{MeV}$ for the electron. The combined electron rest energy becomes 0.5109 $\mathrm{MeV}$, which differs from the measured value by $0.02 \%$.

The numeric value of the elementary charge can be solved for from (13) using $n=-39 / 4$. One obtains $e=1.6021 \mathrm{As}$, which differs from the accepted value by $0.003 \%$.

Changing any one number $i, j, k$ or $l, m, n, p$ by \pm 1 changes the calculated 3D values by factor of 1.26 and 4D (energy) values correspondingly by 1.19 , and the calculated values in Table 2 would be largely incorrect.

The calculated value of the elementary charge differs the least from the measured value. The reason may be the missing of the gravitational constant from the definition of the Planck charge. The gravitational constant is by far the least accurate of the natural constants.

\section{FINE STRUCTURE CONSTANT}

Alpha is defined $\alpha=1 / 2 \mu_{o} e^{2} c / h=2 \pi\left(e / q_{p}\right)^{2}$, which can be written, according to (13), $2 \pi \cdot 2^{-39 / 4}=0.00730$ and $1 / \alpha=137.045$. The difference is $0.007 \%$ with respect to the CODATA value of alpha. In this context, the interpretation of alpha is rather straightforward: The volumetric period doubling process dilutes the initial Planck scale Coulomb energy, and the elementary charge represents the resulting value of the Coulomb energy.

\section{SUMMARY AND CONCLUSIONS}

It is assumed that the electron-positron pair creation is a nonlinear process, and that the periodic structure is simply created by the period doubling phenomenon, which is a universal property of nonlinear dynamical systems. The Planck time, considered as a period, is used as the universal basis for the calculations. 
The numerical calculations in Chapter 2 yield correct values with integer number of doublings without additional parameters or tuning.

The small difference between the calculated and measured values in Table 2 show that the period doubling process is exact after many doublings. This means that the combination of the natural constants used to define the Planck scale quantities has remained constant over time.

The cube root and fourth root differ in that the cube root is positive (or negative), while the fourth root is \pm giving the dipolar property for the elementary charge vs. monopolar mass.

The electron-positron pair is superstable as shown by the observed number of period doublings fulfilling the stability condition.

Considering the electron as a structureless point-like particle excludes the associated periodic structure revealed by this analysis, which shows that the periodic nature of matter also applies to the Coulomb energy of the electric charge and magnetic moment of the electron.

It is therefore concluded that the period doubling process at the Planck scale can be taken as a hypothesis for the origin of the present values of the electron properties and the fine structure constant.

This hypothesis is further corroborated by the good numerical fits in other natural phenomena (e.g. the Cosmic Microwave Background temperature, Hydrogen $21 \mathrm{~cm}$ line, quantized galaxy redshifts [12] and planetary orbits.) [7]. It should be noted that the periodic structure, or intrinsic wave property created by the period doubling process is stationary contrary to the de Broglie wave, which is kinematic.

\section{ACKNOWLEDGMENTS}

I would like to express my special thanks of gratitude to Dr's T. Suntola, H. Sipilä, T. KallioTamminen, M. Hyvönen-Dabek and J. Dabek for many insightful discussions and comments.

\section{REFERENCES}

[1] Rayleigh, L., Maintenance of vibrations by forces of double frequency, and propagation of waves through a medium with a periodic structure, Philosophical Magazine 24: 145-159 (1887).

[2] Martti M. Salomaa and Gary A. Williams, Structure and Stability of Multielectron Bubbles in Liquid Helium, Phys. Rev. Lett. 47, 1730, 14 December 1981. https://doi.org/10.1103/PhysRevLett.47.1730 [3] A. Libchaber, C. Laroche, S. Fauve, Period doubling cascade in mercury, a quantitative measurement, Journal de Physique Lettres, Edp sciences, 1982, 43 (7), pp. 211-216. https//doi.org/10.1051/jphyslet:0198200430702110 0

[4] R. Smolec, I. Soszy' nski, P. Moskalik, A. Udalski, M. K. Szyma' nski, M. Kubiak, G. Pietrzy'nski, Ł. Wyrzykowski, K. Ulaczyk, R. Poleski, S. Kozłowski and P. Pietrukowicz, Discovery of period doubling in BL Herculis stars of the OGLE survey. Observations and theoretical models, Mon. Not. R. Astron. Soc. 419, 2407-2423 (2012). https://doi.org/10.1111/j.13652966.2011.19891.x

[5] T. B. Simpson, J. M. Liu, A. Gavrielides, V. Kovanis, and P. M. Alsing, Period-doubling cascades and chaos in a semiconductor laser with optical injection, Phys. Rev. A 51, 4181, 1 May 1995. https://doi.org/10.1103/PhysRevA.51.4181 [6] Young, D.L., Sheen, H.J. \& Hwu, T.Y. Perioddoubling route to chaos for a swirling flow in an open cylindrical container with a rotating disk. Experiments in Fluids 18, 389-392 (1995). https://doi.org/10.1007/BF00211397

[7] Ari Lehto: On the Planck Scale and Properties of Matter, International Journal of Astrophysics and Space Science. Special Issue: Quantum Vacuum, Fundamental Arena of the Universe: Models, Applications and Perspectives. Vol. 2, No. 6-1, 2015, pp. 57-65. https://doi.org/10.11648/j.ijass.s.2014020601.17 
[8] M. Louis de Broglie: Recherches sur la théorie des Quanta, Ann. Phys., Vol. 10, N³ (1925), pp. 22-128.

[9] Thomson, G. P.: Diffraction of Cathode Rays by a Thin Film, Nature. 119 (3007): 890 (1927).

[10] C. Davisson and L. H. Germer: Diffraction of Electrons by a Crystal of Nickel, Phys. Rev. 30, 705, 1 December 1927.
[11] M. J. Feigenbaum: Universal behavior in nonlinear systems, Physica 7D, (1983), 16-39 [12] William G. Tifft, Redshift - Key to Cosmology, AlphaGraphics, ISBN 978-0-9862619$0-9,2014$ https://williamtifft.wordpress.com/2015/01/26/blog post002-bill-tifft-availability-of-my-book-redshiftkey-to-cosmology-12615/ 